\title{
《O» 5emiп̈г.пеt
}

\section{Introducing Virtual Reality Technologies to Design Education}

\author{
Jonna Häkkilä, Ashley Colley, Jani Väyrynen \\ \& Antti-Jussi Yliharju \\ Faculty of Art and Design, University of Lapland \\ Rovaniemi, Finland \\ Email: jonna.hakkila@ulapland.fi
}

\begin{abstract}
In this paper, we address the introduction of Virtual Reality (VR) tools to the education of industrial design (ID) university students. We present three cases of how we have introduced VR technology in different courses of the industrial design curriculum at the University of Lapland, Finland. As the first example (Case I), we introduced a VR simulation as an empathetic design tool to simulate visual disabilities. The second example (Case II) is reported from a course where students created concepts for a head mounted display (HMD) AR application in smart buildings, and tried out interaction with a HMD VR application. In the third example (Case III), VR was used as a display environment to exhibit students' $3 D$ industrial design concept models. We report our experiences and lessons learnt, as well as recorded student feedback from the trials. As salient findings, we report the general positive feedback, successful integration with the taught themes especially when connected to physical $3 D$ models, as well as suggested improvements. Hindering the adoption of the technology from the teaching point of view, we report on the lack of infrastructure for multi-user groups in classrooms, the additional effort required to set up the technical system, and limited features supporting multimodality.
\end{abstract}

Keywords: Design education, Virtual Reality, Augmented Reality, HeadMounted Displays, Technology in teaching, Media education, Field studies.

\section{Introduction}

Technology is continuously developing at a fast pace, and new application areas that are emerging can potentially change the landscape of how we explore and interact with the world. This constantly developing technology also impacts to education and classroom practices. One of the technologies that is rapidly moving towards mainstream adoption is virtual reality (VR). In virtual reality, a three-dimensional, computer generated environment is created, which can be explored and interacted with by a user. The user is immersed within the digital $3 \mathrm{D}$ world and is able to manipulate objects or perform actions within it. One way to access virtual reality is through a normal computer monitor screen, with early examples being the first-person shooter game, Quake and the Second Life virtual world. Rather than viewing VR on a fixed screen, a higher level of immersion can be achieved by using a head mounted display (HMD), which

(C)2018 (author name/s). This is an Open Access article distributed under the terms of the Creative Commons Attribution 4.0 International License

(http://creativecommons.org/licenses/by/4.o/), allowing third parties to copy and redistribute the material in any medium or format and to remix, transform, and build upon the material for any purpose, even commercially, provided the original work is properly cited and states its license. 
allows the wearer to look around the virtual environment with natural head movement. In addition to the visual sense, virtual reality may be enhanced through other senses such as touch (haptics) and smell.

Virtual and augmented reality technologies have come a long way since their early steps in the beginning of 2000's, when they involved heavy computing devices carried in the user's rucksack and bulky head-mounted display screens, e.g. as used by Cheok et al. (2004) in their human pacman game. Today, VR setups utilize head mounted displays (HMDs) that are lightweight and affordable to large audiences. These are being adopted especially by the entertainment and gaming industries. Research has investigated the use of VR in different domains, including e.g. architecture and museums, and new application areas are appearing. The use of VR in education is an interesting application domain.

Integrating interactive technologies into education already has an extensive history over the past few decades. The first steps in trialing any new technology in education typically occurs through research pilots, but as technologies mature and become commonplace, their adoption becomes easier. This development process can be seen with laptops, mobile phones and tablets, which are today commonplace in schools, realizing the future visions that were trialed e.g. in the Classroom 2000 project two decades ago (Abowd, 1999). These technologies are now common consumer electronics, and their use in education has already been widely investigated. In particular, the use of mobile phones has been extensively researched, ranging from learning to read with inclassroom games (Sanchez et al., 2011) to mobile phone usage as a general classroom learning tool in developing countries (Valderrama et al., 2012). Whereas smartphones are today a mainstream mobile technology, VR still represents a technology of which most students, as well as education professionals, have little or no experience with in practice.

Smartphones and tablets can also function as a platform for augmented reality (AR) and VR applications, and thus they offer a gateway to introduce new technology approaches on a familiar device. Augmented reality, where digital information is overlaid on top of the physical world view, has become a wellknown phenomenon to the youth through the PokemonGo application published in 2016 (Colley et al., 2017). Mobile AR applications have been frequently demonstrated in the context of education, for instance to motivate children to learn about nature on field trips (Chiang et al., 2014; Alakärppä et al., 2017). With virtual reality applications, the user interacts with a digital world which is not visibly linked to the surrounding physical world. Whereas conventional mobile devices, i.e. smartphones and tablets, can be used to access the $3 \mathrm{D}$ virtual world, a higher level of immersion is achieved with head mounted displays (HMDs). HMDs have become the de facto user interface technology for VR, but are still rare enough that the majority of both students and teachers are unfamiliar with their use. The use of HMD VR in education is an underexplored topic, and research investigating its use across different fields of education is lacking. Since VR technologies are rapidly emerging, it is relevant to study their potential in this domain.

In this paper, we address the use of VR, especially HMD based VR, in the area 
of design education. Designers, who create artifacts, applications, and service concepts, for a wide range of end users, have potential to utilize VR technologies for many different purposes. In the following, we first introduce selected related work on the use of VR in education, and then present three case studies, where VR was applied in university level industrial design courses.

\section{Literature Review}

There is a body of meta review papers addressing different aspects of AR \& VR use in education e.g. Bacca et al. (2014), Freina and Ott (2015), Merchant et al. (2014) and Radu (2014). Prior work of specific relevance to our topic falls into the areas of motivation and approach, evaluation of learning outcomes and VR as a student creativity tool.

\section{Motivation and Approach}

Merchant et al. (2014) present a meta review of works applying AR \& VR to education in the K12 age group, concluding that most work on AR and VR in education has focused on different approaches to providing information, e.g. learning capital cities. Further, they report that game-based approaches have yielded better results than those based on simulations or virtual world representations. Considering effectiveness of the mode of use, Merchant et al. (2014) report than individual use outperforms group use, and that in general learning gains deteriorate with repetition, i.e. there is a novelty factor.

The effectiveness of VR simulations as an educational resource is based on their ability to provide: Immersion, Interaction, and User involvement with the environment and narrative (Freina and Ott, 2015). With a focus on simulation, Freina and Ott (2015) report on the different approaches that have been utilised in educational VR research. Four main motivations are identified, visiting inaccessible locations, time travel, experiencing dangerous situations (e.g. firefighter training) and overcoming ethical limitations (e.g. surgery practice) (Freina and Ott, 2015). Further, current applications of VR in education have primarily been applied to adult training or for university students, with relatively little work addressing VR use with younger children (Freina and Ott, 2015).

\section{Evaluation of Learning Outcomes}

Focusing on Augmented Reality, Radu (2014) summarizes benefits that have been identified as improvements in, content understanding, long-term memory retention, physical task performance, student collaboration and student motivation. On the negative side, attention tunneling, usability problems, ineffective integration in the classroom and accommodating learner differences, are reported as challenges to be addressed (Radu, 2014). Based on these findings, a heuristic questionnaire, which may be utilised during the design of educational AR approaches is presented (Radu, 2014). In their meta review of 32 AR education papers, Bacca et al. (2014) summarize that AR has been effective for improving learning performance, learning motivation, student engagement and positive attitudes. On the negative side, limitations of AR are 
reported as: technical performance (e.g. problems locking to markers), learners paying too much attention to virtual information and AR becoming intrusive (Bacca et al., 2014).

\section{VR as a Student Creativity Tool}

Few works have examined the use of VR as a creative tool by students. Kaufmann and Dünser (2007), report on general motivational benefits and ease of use of most AR systems can reduce cognitive load and encourage student exploration and creativity. The use of a collaborative virtual environment by school children is evaluated by Arhippainen et al. (2011), reporting that students were motivated to edit existing content as well as creating new content to the virtual space. Here, also student wishes for private as well as shared space in the virtual world is noted (Arhippainen et al., 2011). Bower et al. (2014), report on a project where students designed AR experiences to augment existing physical sculpture-type artworks, e.g. adding their visual or textual interpretation.

\section{Case Study I - VR Simulation as an Empathetic Design Tool to Simulate Visual Disabilities \\ Introduction}

When approaching a design task, designers aim to "put themselves in the user's shoes", e.g. by conducting end-user research, creating user personas or acting out scenarios in bodystorming sessions. However, in many cases, design is based on recollections of a second person, rather than being experienced firsthand by the designer. This is particularly relevant in the case of disabilities, where it may be difficult for the designer to fully appreciate the issues experienced by disabled users. Focusing on visual impairments, we explored the use of a HMD to enable architects and service designers to gain better end user understanding during architectural and space design processes.

Prior work has shown HMD based visualization provides the most realistic experience during the design phase of buildings, when compared to screen and CAVE formats (Colley et al., 2015). Several works have studied digitally simulated visual impairments in architectural contexts (Ai et al., 2000; Ates et al., 2015; Lewis et al., 2011). Ates et al. (2015) presented an AR HMD solution, where real-world images from a camera were filtered to simulate visual disabilities. VR based solutions, focusing on the visual field loss caused by glaucoma have been presented by Ai et al. (2000) and Jin et al. (2005) in the context of a virtual apartment environment.

\section{Simulating Visual Disabilities}

To explore the potential of HMD based VR as an educational tool for architects and space designers, we created a $3 \mathrm{D}$ virtual environment which designers could navigate via an interface which simulated visual disabilities. Our virtual environment was based on an urban street environment at night, as prior work indicates night vision is particularly problematic for the visually impaired (Klein, 1991). The implementation utilized an Oculus Rift HMD, with an Xbox 360 hand controller used to move around the virtual environment. Four visual 
disabilities were simulated, macular degeneration, cataract, myopia, and glaucoma (see Figure 1). The central symptom of myopia is blurriness, making it difficult to see objects clearly at a distance. Glaucoma often leads to severe loss of sight, and is characterized by tunnel vision. Cataracts result in a clouding of the eye's lens and vision problems are particularly notable in the presence of bright lights. The vision loss caused by macular degeneration is often only partial, and focused on the central area of vision. Details of the implementation of the visual disability filters can be found in Väyrynen et al. (2016).

\section{Evaluation}

Participants were first shown one image the simulated vision of each of the 4 disabilities, which were taken from screenshots of the virtual environment. The participants then put on the HMD and were able to move around the virtual environment using the hand controller. Rather than simply letting the designers to wander through the virtual environment with each visual disability filter active, we devised a navigation task for the test subjects. The subjects were required to navigate along a marked route to checkpoints, where they then had to look around and locate a numeric marker in the scene. The created test software logged the time that it took for the participants to navigate between each checkpoint. Test participants completed the test with normal eyesight and each of the disability filters, the position of the numeric markers being changed between each test case. We evaluated the test set up with 14 design students (8 female), with a mean age of 30 years $(S D=8)$. Participants were encouraged to think aloud during the study. At the end of the test, participants rated both the paper and HMD visualizations' ability to help them better empathize with the visually impaired and value as a design tool, on a 7-point scale.
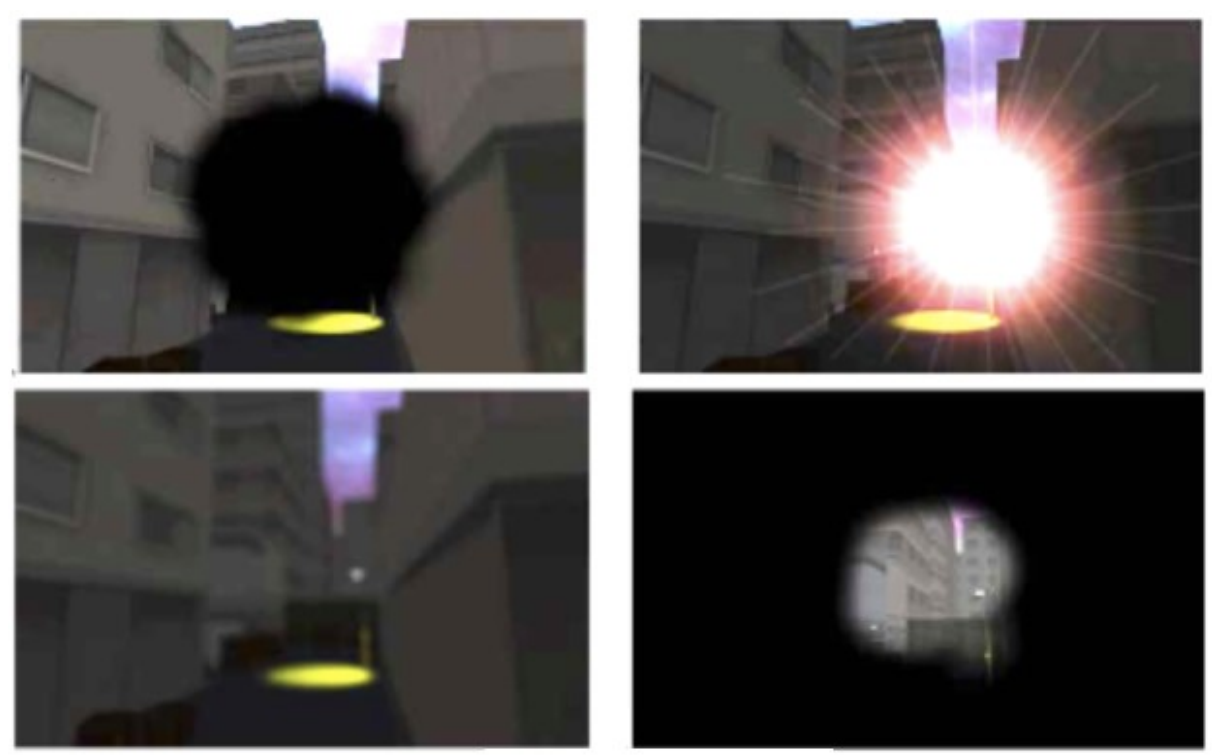

Figure 1. Screenshots from the VR simulation. Top row: macular degeneration, cataract. Bottom row: myopia, glaucoma. Note, brightness of the images has been enhanced for clarity in print. 


\section{Findings}

Compared to the paper visualizations of the visual disabilities, participants considered the HMD VR approach to help them better empathize with the vision impaired (rating 6.3/7 vs. 5.5/7) and to better help design for accessibility (6.0/7 vs. 5.4/7). For example, participants commented, "This is a very illustrative way to identify with visual disabilities. The test was interesting and it was fun to get to try out the head mounted display." (\#3) and "The demo really showed that it is much harder to get around if you have limited vision." (\#1)

No significant differences were found between the times it took participants to complete the test tasks in each condition, however, there were large differences between individual participants. In all the visual disability conditions the participants reported it was more difficult to complete the tasks than in the normal eyesight condition, e.g., "The black spot forced me to move my head in some directions in order to find the labels." (\#12, macular degeneration) and "I can say that when you get closer to signs, especially on a street you cannot really read them, even if you go closer" (\#12, myopia).

Although participants gave positive feedback related to the tool's ability to provide insight to the challenges faced by the visually impaired, the unfamiliar controls of the tool provoked negative comments. Our findings highlight potential issues of unfamiliarity and novelty effects when using VR technology, which need to be overcome before the long-term value of the approach can be identified.

We recognize that simulating disabilities cannot replace the inclusion of disabled users as part of the design process, as noted by Lewis et al. (2011). However, we believe our approach is beneficial in creating empathy with the disabled, and providing designers with some idea of the potential difficulties caused by visual disabilities.

\section{Case Studies II and III - VR for Concept Design and Exhibition for Smart Buildings \\ Design Tasks}

Case studies II and III were integrated to a concept design process, where design students developed new product and application concepts. Virtual and augmented reality technologies were utilized in different phases of the process, and for different purposes in two courses. At the end of both courses a written survey $(n=22, n=11)$ was conducted to collect feedback from the students on their experiences with the technology as part of the learning process. For Case III, the student feedback was complemented with an online survey, to gain deeper insights. The role of virtual and augmented reality technologies in the cases was as follows:

- Trying out a VR HMD to gain an understanding of the technology and to get hands-on experience with it (Cases II and III)

- Creation of a concept design and a low fidelity prototype of a HMD AR application (Case II)

- Creation and modelling of a 3D product design concept, and an exhibit of it in $3 \mathrm{D}$ virtual world (Case III) 
Case study II was conducted as part of the course 'Participatory Design and Interactive Prototypes' at the University of Lapland, Finland, during spring 2017. The course participants $(n=22)$ consisted of bachelor and master's level students from the Industrial Design and Art \& Design study programs. The design task addressed the domain of smart buildings, with the specific application area chosen by the students themselves. For example, students could develop an AR application concept for building maintenance, site electrician, or office worker. The application was to be used with an AR HMD user interface, which the students were asked to demonstrate with a low fidelity prototype (Figure 2.). Low-fi prototypes are non-functional concept prototypes created by using cardboard, paper-cut images and similar materials, as demonstrated e.g. in de Sa \& Churchill, (2012) and Pakanen et al., (2014), and is an often used technique when creating early concept designs of interactive applications. When the same course was run the previous year, the design task had focused on smartphone application concepts. The introduction of HMD VR and AR provided a more futuristic and visionary design challenge for the students.

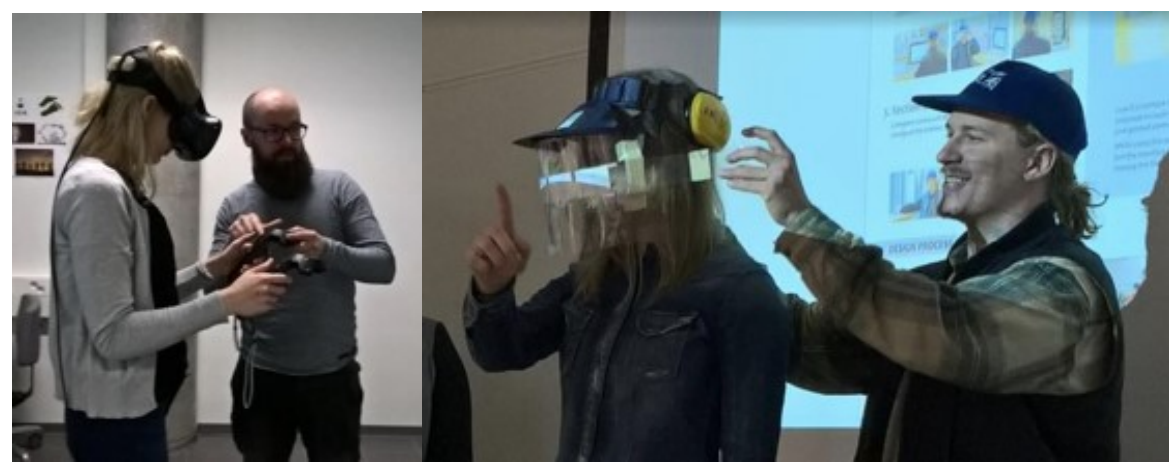

Figure 2. Left: A student trying out a HMD in a classroom setting. Right: Students demonstrating a lo-fi prototype of a head mounted display augmented reality application during a lesson.

Case study III was conducted as part of the course 'Introduction to Concept Design' in spring 2017, where first year bachelor's students studying Industrial Design get their first hands-on touch to product design and the industrial design process. The design task focused on creating an industrial design for a next generation consumer robot, with the specific application area chosen by the student. In addition to the robot's form factor, the students were asked to create a concept and to define the key use cases, use context, and to explore the design from the human-robot interaction point of view. During the course, the software packages Blender and Google Tilt Brush were used for $3 \mathrm{D}$ modelling and visualization tasks. The created concept designs were then $3 \mathrm{D}$ printed, to create physical models and the virtual models were imported to the Unity $3 \mathrm{D}$ software environment to create a virtual exhibition space. At the end of the course, a public exhibition was organized. Here, the virtual $3 \mathrm{D}$ design models were exhibited together with the physical models, design posters, and packaging designs (Figure 3). During the exhibition, the virtual world presentation could be viewed through a HMD, as well as with a laptop computer. In previous years, the course had focused on common everyday life objects. Now, the students 
were encouraged to create visionary product designs concepts and also consider the required support material for brand development, new markets and emerging technologies. The use of VR aligned well with these targets.

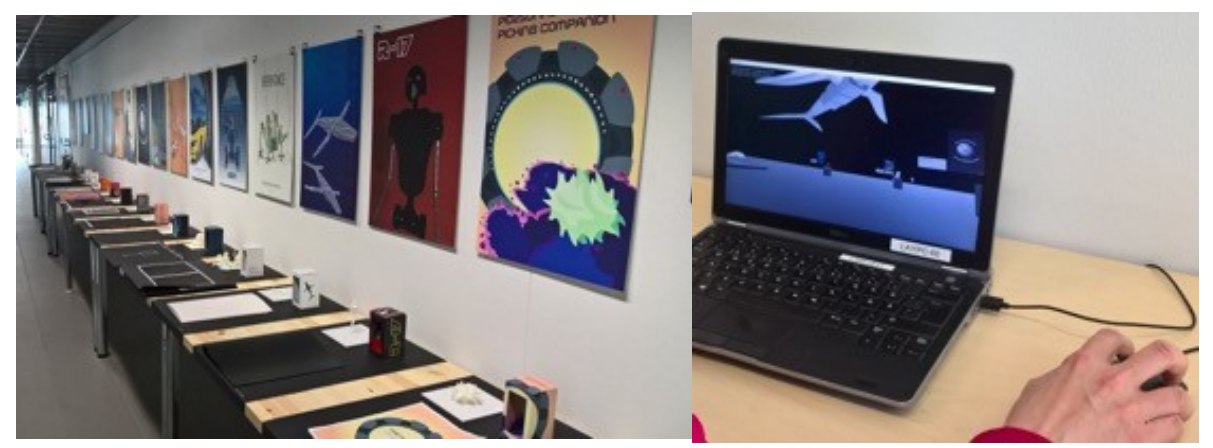

Figure 3. Left: Exhibition of students' 3 D printed physical concepts, packaging concepts and posters. Right: The students' designs in a virtual reality exhibition space viewed with a laptop computer.

\section{Student Feedback}

The feedback from students on the use of virtual reality techniques and HMDs fell into two different categories, hedonic and utilitarian. Firstly, the use of the technologies was perceived with hedonic aspects, i.e. inspiring, delightful, fun, and providing novel and exciting experiences. Secondly, the approach was seen as having utilitarian benefits in improving the understanding the design challenges as well as, in the case of $3 \mathrm{D}$ models, the students seeing their own designs from alternative standpoints.

In Case study III, when the students explored their own 3D modelled designs in a virtual world through a HMD, the feedback received focused on three themes: scale, realism, and impressive presentation. Also, seeing one's own design in a virtual world, after looking at it for a long time on paper, was commented as a motivating experience, bringing new motivation, perspective and excitement to the design task. Using a HMD helped students to better understand the scale and physical dimensions of their designs, as illustrated in the following comments: "[It was important to view the models with a HMD] as it helped to understand the measures and perspectives, I feel I improved all the time" (student \#1), "It gave me a better perspective about the size of my design. It was a great experience" (\#6). The HMD was seen as a valuable tool when presenting one's design, e.g. "The presentation and viewing experience crowns the design work." (\#5). The level of immersion was also mentioned adding to the experience with the design: "With virtual glasses, I could immerse into the environment much better and the experience was much more impressive" (\#9).

Generally, in both Case II and III, using VR and HMD was seen as the way of the future, and using them as part of the design process was seen as a motivating experience. "[I felt] it took my work to an entirely new level. This is the future" (Case III, student \#2). In both design tasks, it was perceived as motivating to get an introduction to using future technology. Using VR and HMDs were appreciated as new tools that added to the student's skill set. In Case study II, the VR and AR exercises were seen to provide insight to different methods of 
prototyping and participatory design, and in applying low-fi prototyping to different domains.

\section{Discussion and Conclusions}

In this paper, we have presented three case studies, where virtual and augmented reality technologies have been integrated into the university level education of industrial design students. The case studies have covered the use of VR as an empathetic design tool, as well as using the technology in different parts of the design process: in concept creation, in the prototyping phase, and for exhibiting a final design. Virtual reality and the use of head-mounted displays were found to offer both inspiring and motivating experiences, as well as to function as a utilitarian tool in the design process. As all our participants were first time VR users, the novelty effect should be considered when evaluating the findings. Thus, it is not apparent if the tools would be as motivating in repeated use. Prior works such as Merchant et al. (2014) and Bacca et al. (2014) have noted that the novelty effect is strong with VR solutions.

The three case studies resulted in generally very positive feedback from students about integrating VR technologies as part of the courses. Enthusiasm for the stimulating experiences were clearly observed, and also expressed in written feedback from the students. The experience rich approach of setting the design task (Case II), in gaining end-user insight (Case I), and in viewing one's own designs (Case III) were appreciated. As reported by Bower et al. (2014), our Case III demonstrates the potential to introduce VR as an extension to conventional learning tasks.

Our experiences in the case studies also provided insight about factors that are hindering the adaptation of the technology from the teaching point of view. The lack of infrastructure for multi-user groups in classrooms sets limits for the practicality of the tasks, as for instance in our case, the whole student group was using only one set of VR equipment. Here, our findings echo those of Merchant et al. (2014), who report that VR solutions generally suit individual use. In this respect, rather than each student being in their own individual virtual world, we believe solutions should aim towards a common virtual environment, such as that demonstrated by Greenwald et al. (2017). Also, and again in line with Merchant et al. (2014), we note the effort required to set up the technical system required extra time when preparing the lessons, and this may limit the technology use in classroom teaching. However, such factors can be expected to reduce in the future as the technology becomes more commonplace, similarly to issues associated with the introduction of language laboratories to schools in the 1970 s.

Finally we note the limited features supporting multimodality affect to the possibilities when concepting and prototyping VR applications, as well as creating and experiencing a virtual design exhibitions. Altogether, there is still much to develop in creating easy and flexible tools for VR in design education. However, with our case studies we can conclude that VR technologies can already be successfully integrated to industrial design education, where they offer interesting and useful possibilities. 


\section{Acknowledgements}

This work was funded by Business Finland as part of the VARPU project and by the European Social Fund in the Design or Die project. 


\section{References}

Abowd, G. D. (1999). Classroom 2000: An experiment with the instrumentation of a living educational environment. IBM systems journal, 38(4), 508-530.

Ai, Z., Gupta, B. K., Rasmussen, M., Lin, Y. J., Dech, F., Panko, W., \& Silverstein, J. C. (2000). Simulation of eye diseases in a virtual environment. In Proceedings of the 33rd Annual Hawaii International Conference on System Sciences (p. 5 pp.-). https://doi.org/10.1109/HICSS.2000.926803

Alakärppä, I., Jaakkola, E., Väyrynen, J., \& Häkkilä, J. (2017). Using nature elements in mobile AR for education with children. In Proceedings of the 19th International Conference on Human-Computer Interaction with Mobile Devices and Services (MobileHCI '17). ACM, New York, NY, USA, Article 41, 13 pages. DOI: https://doi.org/10.1145/3098279.3098547

Arhippainen, L., Pakanen, M., Hickey, S., and Mattila, P. (2011). User experiences of 3D virtual learning environment. In Proceedings of the 15th International Academic MindTrek Conference: Envisioning Future Media Environments (MindTrek '11). ACM, New York, NY, USA, 222-227. DOI: https://doi.org/10.1145/2181037.2181075

Ates, H. C., Fiannaca, A., \& Folmer, E. (2015). Immersive Simulation of Visual Impairments Using a Wearable See-through Display. In Proceedings of the Ninth International Conference on Tangible, Embedded, and Embodied Interaction (pp. 225228). New York, NY, USA: ACM. https://doi.org/10.1145/2677199.2680551

Bacca, J., Baldiris, S., Fabregat, R., \& Graf, S. (2014). Augmented reality trends in education: a systematic review of research and applications. Journal of Educational Technology \& Society, 17(4) 133.

Bower, M., Howe, C., McCredie, N, Robinson, A., and Grover, D. (2014). Augmented Reality in education-cases, places and potentials. Educational Media International 51, 1: 1-15.

Cheok, A. D., Goh, K. H., Liu, W., Farbiz, F., Fong, S. W., Teo, S. L., ... \& Yang, X. (2004). Human Pacman: a mobile, wide-area entertainment system based on physical, social, and ubiquitous computing. Personal and ubiquitous computing, $8(2), 71-81$.

Chiang, T. H., Yang, S. J., \& Hwang, G. J. (2014). An augmented reality-based mobile learning system to improve students' learning achievements and motivations in natural science inquiry activities. Journal of Educational Technology \& Society, $17(4), 352$.

Colley, A., Väyrynen, J., \& Häkkilä, J. (2015). Exploring the Use of Virtual Environments in an Industrial Site Design Process. In Human-Computer Interaction - INTERACT 2015 (pp. 363-380). Springer, Cham. https://doi.org/10.1007/978-3-319-22723-8_29

Colley, A., Thebault-Spieker, J., Lin, A. Y., Degraen, D., Fischman, B., Häkkilä, J., ... \& Wenig, D. (2017, May). The geography of Pokémon GO: beneficial and problematic effects on places and movement. In Proceedings of the $2017 \mathrm{CHI}$ Conference on Human Factors in Computing Systems (pp. 1179-1192). ACM.

Freina, L., \& Ott, M. (2015). A literature review on immersive virtual reality in education: state of the art and perspectives. In The International Scientific Conference eLearning and Software for Education (1)133-141.

Greenwald, S. W., Corning, W., \& Maes, P. (2017). Multi-User Framework for Collaboration and Co-Creation in Virtual Reality. 12th International Conference on Computer Supported Collaborative Learning (CSCL). 
Jin, B., Ai, Z., \& Rasmussen, M. (2005). Simulation of eye disease in virtual reality. In Engineering in Medicine and Biology Society, 2005. IEEE-EMBS 2005. 27th Annual International Conference of the) pp. 5128-5131). IEEE.

Kaufmann H., \& Dünser, A. (2007) Summary of Usability Evaluations of an Educational Augmented Reality Application. In: Shumaker R. (eds) Virtual Reality. ICVR 2007. Lecture Notes in Computer Science, vol 4563. Berlin, Heidelberg: Springer,

Klein, R. (1991). Age-Related Eye Disease, Visual Impairment, and Driving in the Elderly. Human Factors, 33(5), 521-525. https://doi.org/10.1177/001872089103300504

Lewis, J., Brown, D., Cranton, W., \& Mason, R. (2011). Simulating visual impairments using the Unreal Engine 3 game engine. In 2011 IEEE 1st International Conference on Serious Games and Applications for Health (SeGAH) (pp. 1-8). https://doi.org/10.1109/SeGAH.2011.6165430

Pakanen, M., Colley, A., Häkkilä, J., Kildal, J., \& Lantz, V. (2014). Squeezy bracelet: designing a wearable communication device for tactile interaction. In Proceedings of the 8th Nordic Conference on Human-Computer Interaction: Fun, Fast, Foundational (pp. 305-314). ACM.

De Sá, M., \& Churchill, E. (2012). Mobile augmented reality: exploring design and prototyping techniques. In Proceedings of the 14th international conference on Human-computer interaction with mobile devices and services (pp. 221-230). ACM.

Merchant, Z., Goetz, E. T., Cifuentes, L., Keeney-Kennicutt, W., \& Davis, T. J. (2014). Effectiveness of virtual reality-based instruction on students' learning outcomes in K-12 and higher education: A meta-analysis. Computers \& Education, 70, 2940 .

Radu, I (2014). Augmented reality in education: a meta-review and cross-media analysis. Personal and Ubiquitous Computing, 18(6), 1533-1543.

Sánchez, I., Cortés, M., Riekki, J., \& Oja, M. (2011, June). NFC-based interactive learning environments for children. In Proceedings of the 10th international conference on interaction design and children (pp. 205-208). ACM.

Valderrama Bahamóndez, E., Häkkilä ,J., \& Schmidt, A. (2012). Towards better UIs for mobile learning: experiences in using mobile phones as multimedia tools at schools in rural Panama. In Proceedings of the 11th International Conference on Mobile and Ubiquitous Multimedia (p. 39). ACM.

Väyrynen, J., Colley, A., \& Häkkilä, J. (2016). Head mounted display design tool for simulating visual disabilities. In Proceedings of the 15th International Conference on Mobile and Ubiquitous Multimedia (pp. 69-73). ACM. 